\title{
LES FORCES HYDRAULIQUES DE LA HONGRIE
}

\section{E. MOSONYI}

Chef du Service Hydroélectrique du Ministère Hongrois de I'Agriculture

La première statistique détaillée se rapportant aux forces hydroélectriques disponibles de la Hongrie fut publiée en 1905 (1). Cette œurre basée sur des levés hydrologiques et topographiques détaillés ainsi que sur des observations limniméiriques de plusieurs années totalisant une cenlaine de jaugeages était, er son genre, une ouvre de pionnier. En effet, ce ne fut qu'à cette époque que l'on a commencé, en France, les levés systématiques relatifs aux forces hydrauliques; quant aux statistiques de la Suisse el de la Norvège, elles ne sont pas antérieures à l'ounve de Viczian.

La multitude de données et les indications utiles que comporte l'œuvre de Viczran rendent encore à l'heure actuelle de bons services à tous ceux qui s'intéressent aux forces hydrauliques du bassin des Carpates; c'est ainsi qu'elle a servi de point de départ aux statistiques récenles de la Roumanie et les Tchécoslovaques l'ont également utilisée. Cette cuvre, cependant, ne donne pas de renseignements en ce qui concerne les ressources actuelles d'énergie hydraulique du pays, car les statistiques suivant l'habitude technique et économique d'alors, ont négligé l'énergie des secteurs de plaine des rivières: et pourtant la Hongrie actuelle n'a que ces secteurs.

Les statistiques publiées depuis 1920 , établies plus ou moins arbitrairement, ne peuvent être regardées comme complètes. Par conséquent, à l'heure actuelle, comme les besoins en énergie électrique vont toujours en augmentant, les ressources hydroélectriques intéressent de plus en plus les milieux compétents; aussi le Service Hydroélectrique procéda d'urgence à la détermination du potentiel hydroélectrique du pays. En effet, ce n'est que sur la connaissance exacte de ce potentiel hydroélectrique qu'on pourra déterminer conformément au degré de développement

(1) E. Viczun : "Magyarorszag vizierol »-Les Forces Hydrauliques de la Hongrie - 2e Edition 1913. de la science, d'une part la lotalité de l'énergie utilisable et, d'autre part, l'intérêt économique présenté par l'énergie pouvant être utilisée d'une manière rationnelle.

Le potentiel d'énergie d'une rivière ou d'un système de cours d'ean est donné par la formule :

$$
\mathrm{P}=\mathrm{\Sigma} \frac{1000 q h}{75} 0.736=9.8 \mathrm{\Sigma} \mathrm{qh} \ldots \mathrm{kw}
$$

en additionnant l'énergie des secteurs successifs, où $\ll q »$ désigne le débit du secteur délimité par deux affuents successifs en $\mathrm{m}^{3} / \mathrm{sec}$. et « $h »$ désigne la dénivellation enire les deux points extrêmes dudit secteur.

Le débit étant variable, le potentiel d'énergie peut être caraciérisé de plusieurs façons. Aupa-

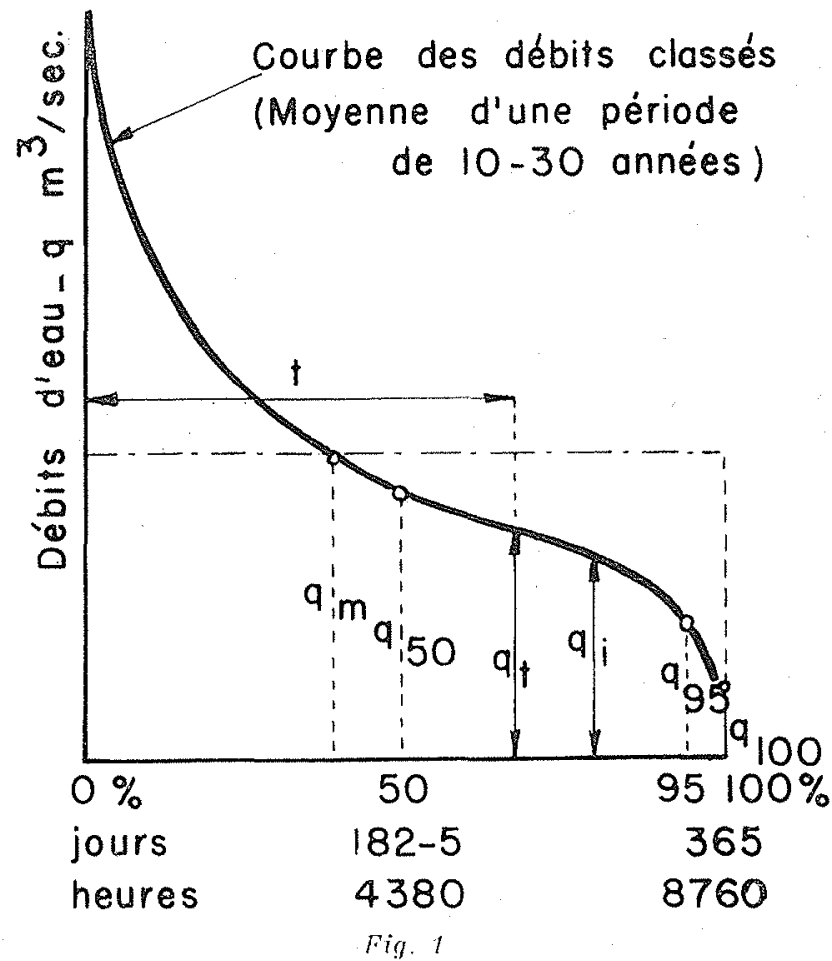


ravant on a détermine -... comme c'est le cas dans l'ouvre de VIczidx - Ia puissance theorique de $100 \%$ correspondant at minimum te débit, done la puissance disponible d'une manière constante, tandis qu'à l'heure actuelle on indique sur la base de la courbe des débits classés les puissances $\mathrm{P}_{4: 3}$ et $\mathrm{P}_{\text {: }}$ déterminées moyennant des débits $q_{1 !: ;}$ et $q_{: 00}$ correspondant aux durées de $95 \%$ (fig. 1) et de $50 \%$ (fig. 1) d'une année.

La Conférence Mondiale d'Energie a adoptó également l'indication simuliance de ces deax domées pour la base de la statisticue mondiale d'énergie hydraulique.

Il va de soi qu'on n'obtient pas de valeurs exactes de $\langle P\rangle$ en se servant de la courbe des débits classés correspondant à une période suffisamment longue. En effet, la variation du débit modifie la chute $h »$, c'est donc uni- quement la courbe de durée des puissances qui domnerait théoriquement les résultats exacts. On se passe, cependant, dans la pratique, de la construction de cette courbe.

La valeur économique du potentiel hydroélectrique est influencée par de nombreux facteurs : situation géographique, conditions topographiques et géologiques, possibilités de mise en valeur du courant produit, etc... Mais, au point de vue purement hydrologique, les facteurs exercant une influence décisive sur celte valeur sont les suivants : ble.

a). La valeur absolue de la puissance disponi-

b). L'importance relative du débit ; puisqu'on peut obtenir la mème puissance en utilisant un clébit faible et une grande chute ou bien un gros débit et une chute réduite, au point de vue éco-

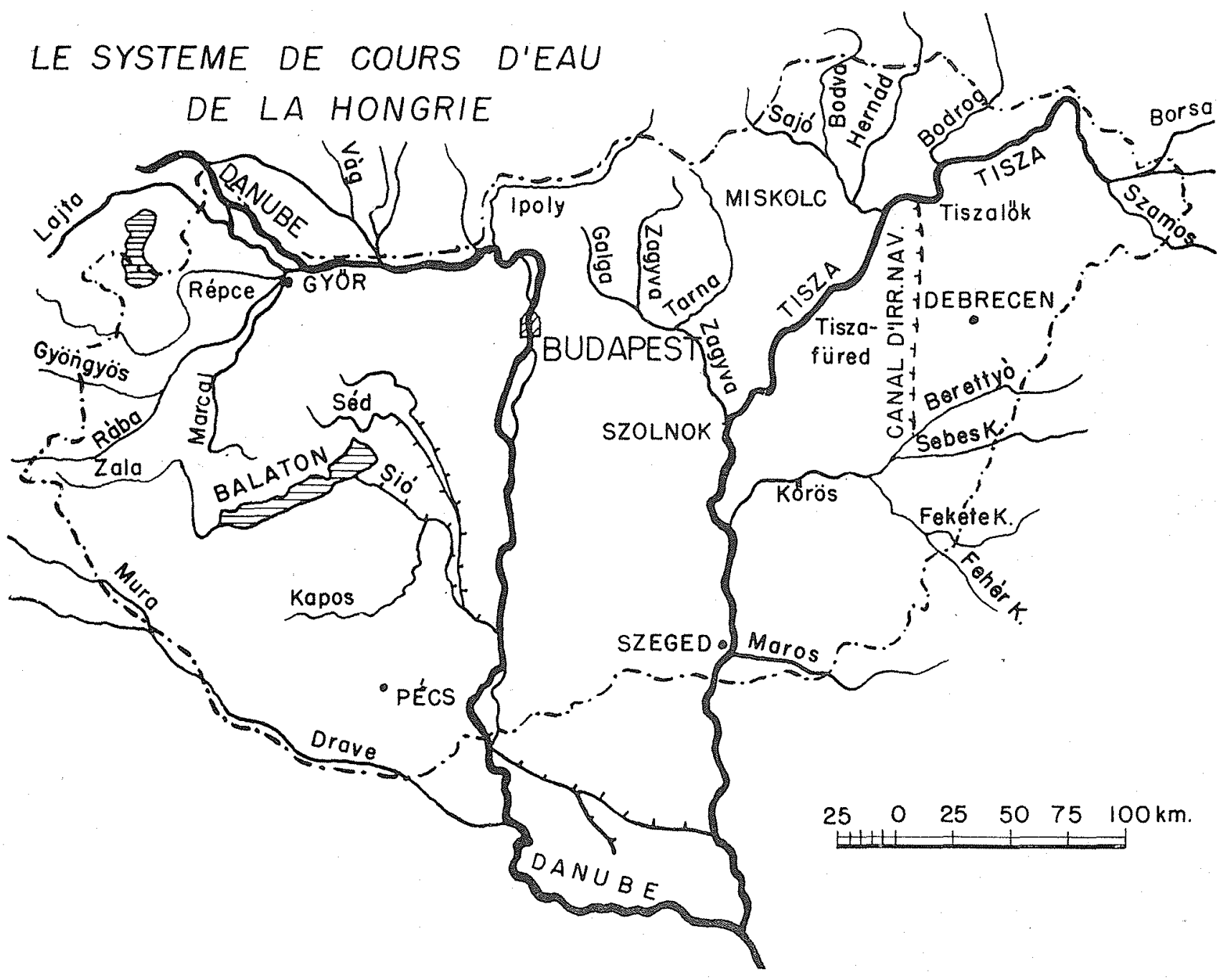

Fig. 2 


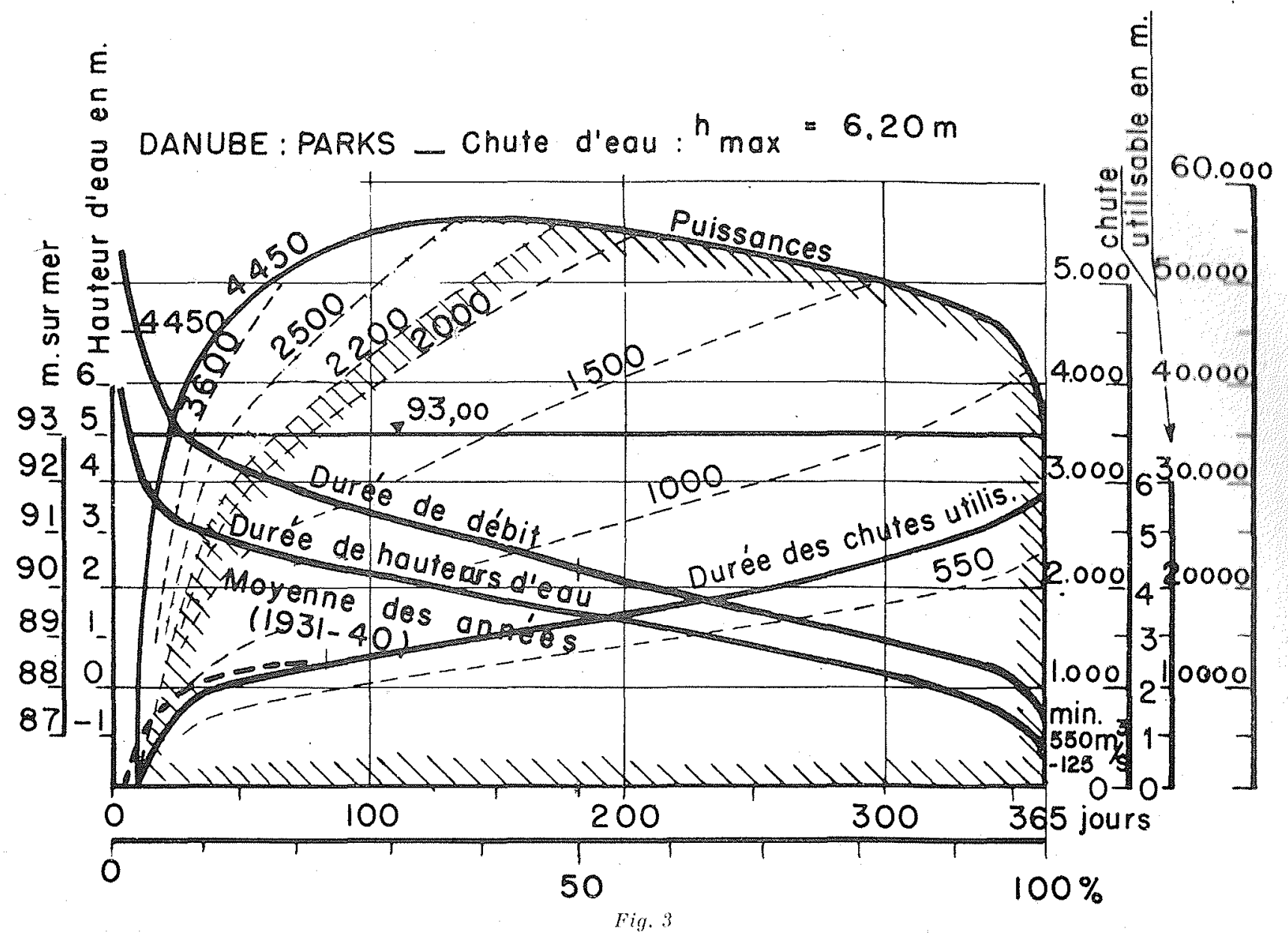

nomique, le premier cas est plus favorable. De ce fait, les forces motrices des régions montagneuses offrent, en général, plus d'avantages.

c). La variabilité relative de la paissance disponible, qui peut etre caractérisée par le rapport :

$$
\alpha=\frac{P_{30}}{P_{0 ; 0 ;}} \text { on } \alpha_{1}=\frac{P_{50}}{P_{1(i)}}
$$

Plus cette valeur est petite, plus les conditions sont favorables.

Suivant les recherches poussées

$$
\alpha_{1}=4 \cdot 0
$$

dans le eas du Danube hongrois,

$$
\alpha_{1}=1 \cdot 0 \ldots 6 \cdot 0
$$

pour les rivieres de la région occidentale du pays,

$$
\begin{aligned}
& \alpha_{1}=6 \% \text { pour la Tisza et } \\
& \alpha_{1}=7-11 \cdot 0 \text { pour ses aflluents, }
\end{aligned}
$$

valeurs dans lesquelles s'expriment les propricm
Lés caractéristiques des bassins versants et les conditions climatiques.

Les valeurs « $\alpha \gg$ ou $\ll \alpha_{1} »$ permeltent de tirer des conclusions relatives aux puissances disponibles des rivières, dont les conditions hydrologiques sont peu étudiées.

Le potentiel hydroélectrique peut ètre également apprécié par les quantités d'énergie disponibles $E_{100} \quad E_{9: 3} \quad E_{50} / \mathrm{kwh} /$, valeurs correspondant aux parties respectives de l'aire délimitée par la courbe de durée des puissances.

Le relevé ci-dessous peut être considéré comme provisoire car, en ce qui concerne les cours d'eau de moindre importance, on ne dispose pas encore d'un nombre suffisant de jaugeages. Malgré cela, la valeur totale du potentiel d'énergie est sensiblement exacte puisque le Danube et la Tisza suffisamment étudiés au point de vue hydrométrique représentent plas de $3 / 4$ de nos ressources hydroélectriques.

Il y a lieu, d'ailleurs, de signaler que les cal- 


\section{DANUBE:PAKS MOYENNE DES ANNEES 1931-1940}

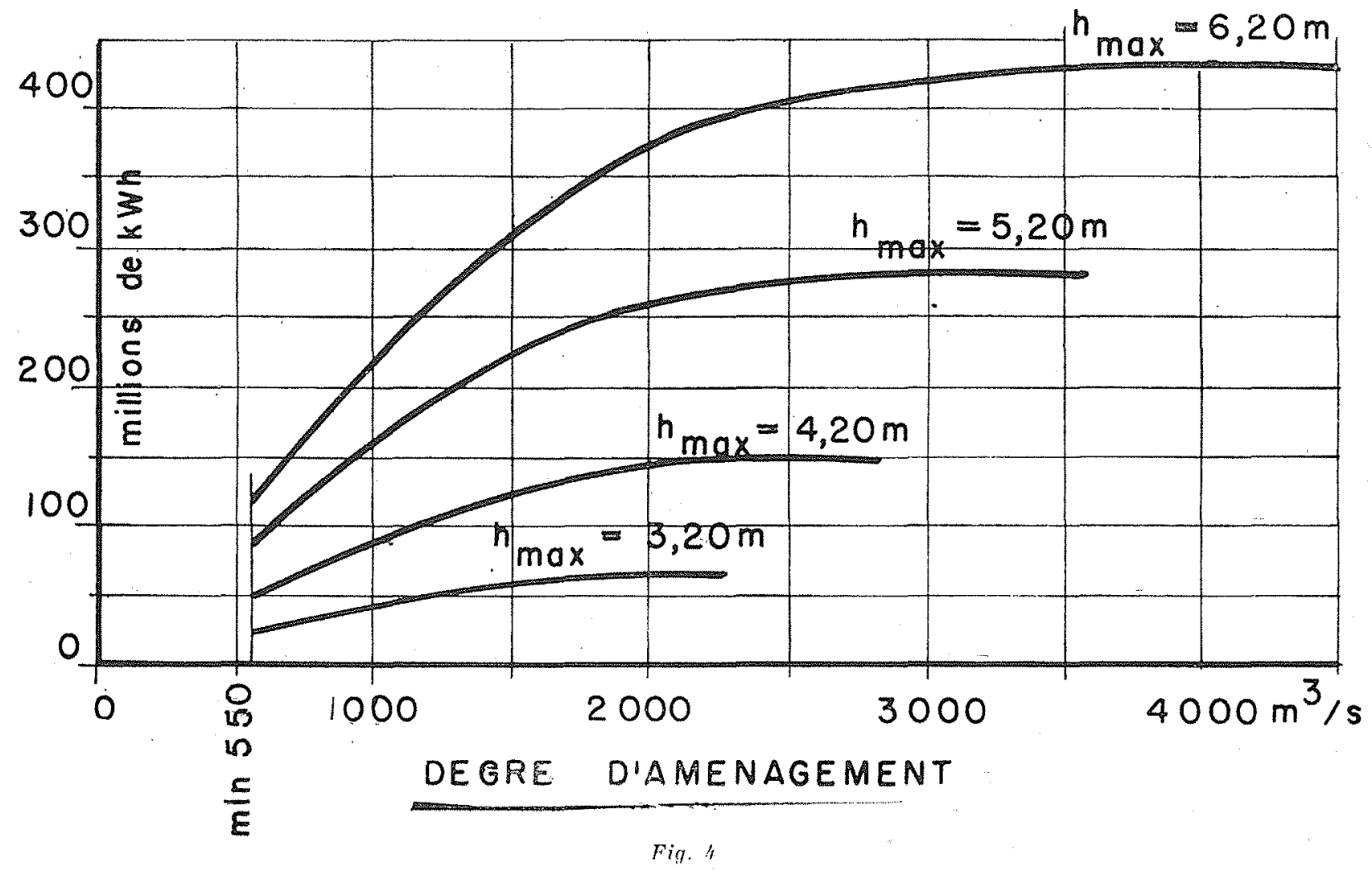

culs ont été effectués en se basant sur le total du débit, abstraction faite de diverses exigences autres que celles de l'utilisation des forces motrices.

Sur la base des données disponibles, il nous a semblé plus exact de déterminer les puissances de durée de $100 \%$ correspondant au minimum de débit. En raison de cela, au lieu des valeurs $P_{9:} \quad$ figurant, de préférence, dans les statistiques internationales, il y a lieu de signaler que pour la Hongrie le rapport $P_{9: 3} / P_{\text {to }}$ est de 1.5 à 2.0 .

En ce qui concerne les cours d'eau formant frontière du pays, nos données n'indiquent que la moitié de la puissance disponible.

En calculant les énergies, nous avons remplacé la fig. délimitée par la courbe de durée par une fig. rectiligne, ce qui veut dire :

$\mathrm{E}_{0,0}=4880 \mathrm{P}_{00}+\frac{\mathrm{P}_{30}+\mathrm{P}_{103}}{2} \mathrm{kwh}$, tandis que

$\mathrm{E}_{\mathrm{tow}}=8760 \mathrm{P}_{\mathrm{t}(\mathrm{H})} \ldots \ldots \mathrm{kwh}$.
TABLEAU I

Le potentiel d'énergie hydraulique de la Hongrie $[\mathrm{P}=9.8 \quad \mathrm{q} h \ldots \ldots \mathrm{kw}]$

\begin{tabular}{|c|c|c|c|c|c|}
\hline SYSTEME & DU & ANUBE & & & \\
\hline $\begin{array}{c}\text { Cours d'eau et sectemr } \\
\text { du fleuve ( } \mathrm{r})\end{array}$ & $\begin{array}{l}P_{100} \\
r .000 \\
k w w\end{array}$ & $\begin{array}{l}\mathrm{E}_{100} \\
\mathrm{r} .000 \\
\text { kwh }\end{array}$ & $\begin{array}{c}P_{30} \\
\substack{t .000 \\
k w}\end{array}$ & $\begin{array}{l}\mathrm{E}_{\mathrm{ij} \theta} \\
\text { mill. } \\
\text { kwh }\end{array}$ & $\frac{\mathrm{E}_{i 00}}{\sum_{\%} \mathrm{E}_{00}}$ \\
\hline 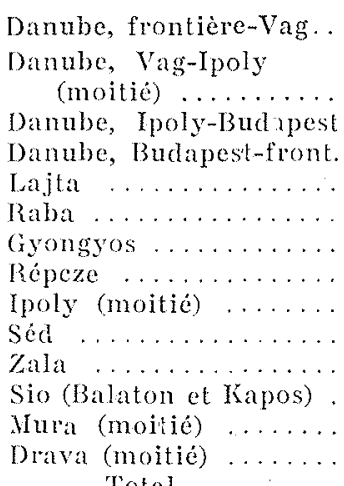 & \begin{tabular}{r|}
0.7 \\
24.3 \\
82.5 \\
0.6 \\
9.6 \\
0.9 \\
0.7 \\
0.1 \\
0.3 \\
0.7 \\
0.2 \\
5.4 \\
14.8 \\
\end{tabular} & $\begin{array}{r}85.0 \\
213.0 \\
724.0 \\
5.3 \\
84.1 \\
7.9 \\
6.1 \\
0.9 \\
2.6 \\
6.1 \\
1.8 \\
46.9 \\
130.0 \\
\end{array}$ & $\begin{array}{r}40.0 \\
107.0 \\
340.0 \\
2.8 \\
47.9 \\
5.2 \\
3.9 \\
1.9 \\
1.8 \\
4.2 \\
6.8 \\
21.4 \\
63.0 \\
\end{array}$ & $\begin{array}{r}285.0 \\
756.0 \\
2.420 .0 \\
19.7 \\
336.0 \\
37.6 \\
29.0 \\
12.7 \\
12.4 \\
29.2 \\
57.6 \\
152.0 \\
445.0 \\
\end{array}$ & \begin{tabular}{|r|}
21.3 \\
3.9 \\
10.1 \\
32.8 \\
0.3 \\
4.5 \\
0.5 \\
0.4 \\
0.2 \\
0.2 \\
0.4 \\
0.8 \\
2.1 \\
6.0 \\
0.1
\end{tabular} \\
\hline
\end{tabular}

(1) Voir fig. 2. 


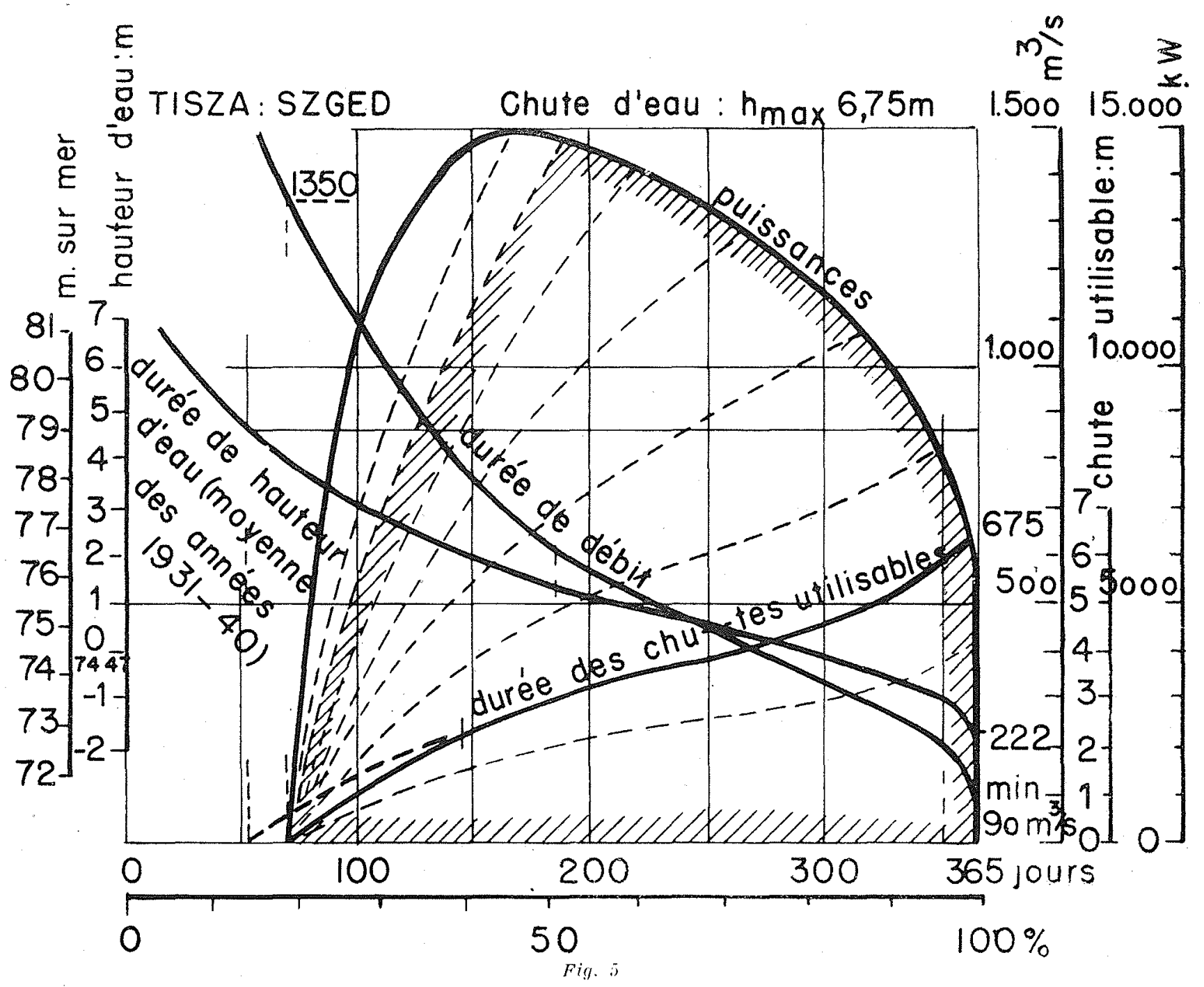

\begin{tabular}{|c|c|c|c|c|c|}
\hline \multicolumn{6}{|c|}{ SYSTEME DE LA TISZA } \\
\hline $\begin{array}{l}\text { Cours d'eau et sectenr } \\
\text { dis fleuve }\end{array}$ & $\begin{array}{c}P_{100} \\
\substack{\text { r.000 } \\
\mathrm{kw}}\end{array}$ & $\begin{array}{c}\mathrm{E}_{100} \\
\text { mill. } \\
\mathrm{kwhil}\end{array}$ & $\begin{array}{c}P_{30} \\
\text { r.opo } \\
k w\end{array}$ & $\begin{array}{c}\mathrm{E}_{300} \\
\text { mill1. } \\
\text { kwh }\end{array}$ & $\frac{\mathrm{E}_{; j ; 0}}{\sum \mathrm{E}_{i ; 0}}$ \\
\hline Tisza, frontière-Borsa & 1.8 & 15.7 & 11.2 & 77.5 & 1.1 \\
\hline Tisza, Boéza-Szamo & 2.0 & 17.8 & 12.9 & 89.2 & 1.2 \\
\hline Tisza, Szamos-Bodrog & 5.3 & 46.4 & 33.5 & 232.0 & 3.2 \\
\hline Tisza, Bodrog-Sajo & 1.0 & 8.6 & 6.2 & 42.9 & 0.6 \\
\hline l'isza, Sajo-Zagyva & 5.7 & 50.0 & 36.2 & 251.0 & 3.4 \\
\hline Tisża, Zagyva-Koros & 2.2 & 19.5 & 14.0 & 97.5 & 1.3 \\
\hline Tisza, Koros-Maros & 1.0 & 8.8 & 6.4 & 44.4 & 0.6 \\
\hline Tisza, Maros-front & 0.6 & & 4.0 & 27.6 & 0.4 \\
\hline Szamos ........ & 1.2 & 10.9 & 7.5 & 51.8 & 0.7 \\
\hline Bodrog & 0. & 1. & 1. & 7.5 & 0.1 \\
\hline Sajo. & 1.8 & 15.8 & 14.2 & 97.1 & 1.3 \\
\hline Bodva. & 0.5 & 4.5 & 3. & 23.4 & 0.8 \\
\hline Hermad. & 2.3 & 20.5 & 14.1 & 98.0 & 1.3 \\
\hline Zagywa (Galga, Tarna & 0.4 & 3.3 & 3.8 & 25.4 & 0.3 \\
\hline Les lioros . . . . . . & 0.8 & 7.0 & 7.6 & 51.7 & 0.7 \\
\hline Total & 26.8 & $236.0 !$ & 176.2 & 1217.0 & 16.5 \\
\hline Hongrice, ant lotal & 230.1 & 2018.71 & $\mid 043.6$ & 7382.2 & $\overline{100.0}$ \\
\hline
\end{tabular}

Le résultat final est que le potentiel hydroélecirique de la Hongrie, en se basant sur les débits de $50 \%$ de durée, se chiffre par 1,1 million de kw soit 7.500 millions de kwh.

Suivant la dernière colonne du tableau, les syslèmes du Danube et de la Tisza représentent respectivement les $68 \%$ et $12 \%$ du potentiel hydroélectrique total du pays. La diversité hydrologique de ces deux systèmes de fleuve s'exprime, d'ailleurs, par le rapport $\mathrm{E}_{; 0} / \mathrm{E}_{100}$ qui est respectivement de 3.5 et 6.0 pour le Danube et la Tisza.

En faisant une comparaison entre les données hongroises et celles relatives à la statistique mondiale (tableau II) il en ressort que le potentiel spécifique de la Hongrie est de $3.4 \mathrm{cv} / \mathrm{km}^{2}$ presctre identique it la moyenne relative du 
TABLEAU II

Bases de calcul $:$ débit $=q_{9 ;}$ rendement $=70 \%$

\begin{tabular}{|c|c|c|c|c|c|c|}
\hline \multirow[b]{2}{*}{ Continent } & \multicolumn{3}{|c|}{ HYDRAULIQUES } & \multicolumn{3}{|c|}{ DU MONDE } \\
\hline & $\begin{array}{l}\text { Putiss } \\
\text { théor } \\
\text { mil } \\
\text { millio } \\
\mathrm{CV}\end{array}$ & $\begin{array}{l}\text { mess } \\
\text { maes } \\
\text { s de } \\
\%\end{array}$ & $\begin{array}{c}\text { Super } \\
\text { ficie } \\
\text { mill. } \\
\text { lims }\end{array}$ & $\begin{array}{l}\mathrm{CV} \\
\mathrm{km}=\end{array}$ & $\begin{array}{l}\text { Enabi- } \\
\text { tants } \\
\text { en } \\
\text { millions }\end{array}$ & $\begin{array}{l}\mathrm{CV} / \\
\text { miil. } \\
\text { ancs }\end{array}$ \\
\hline Europe & 56 & $1 \overline{3}$ & 10 & 5.6 & 531 & 105 \\
\hline$A \operatorname{sic}^{*} \ldots \ldots \ldots$ & 71 & 16 & 41 & 1.7 & 1.198 & 59 \\
\hline Américg du Nord & 69 & 15 & 24 & 2.9 & 180 & 383 \\
\hline Améric. du Sud & 44 & 10 & 18 & 2.4 & 93 & 473 \\
\hline Afrique $\ldots . .$. & 190 & 42 & 30 & 6.3 & 157 & 1.210 \\
\hline Australie et $O c$ & 17 & 4 & 9 & 1.9 & 11 & $1.54 i$ \\
\hline Le monde entier & 447 & 100 & 132 & 3.4 & 2.170 & 206 \\
\hline
\end{tabular}

monde, tandis que les $3.4 \mathrm{cv}$ revenant à 1.000 habitants sont bien inférieurs à la moyenne mondiale : $206 \mathrm{cv} / 1.000$ àmes, mème aux $105 \mathrm{cv}$ de l'Europe, sans parler, par exemple, des 1.400 $\mathrm{ev} / 1.000$ habitants de la Suisse.

Il va de soi que ce n'est qu'une partic du potenliel hydroelectrique se chiffrant par 1.1 million $\mathrm{kw}$, soit 7.500 millions de $\mathrm{kwh}$, qui peut être ulilisée. En dehors des pertes dues à la pente des canaux d'amenée et de fuite ou - dans le cas de canalisation de rivières - aux courbes de remous, enfin à la transformation de l'énergie, il faut considérer qu'on n'est pas à mème d'exploiter la puissance de certains secteurs (du fait des conditions d'agglomération, etc...) et enfin on ne doit pas oublier qu'en cas

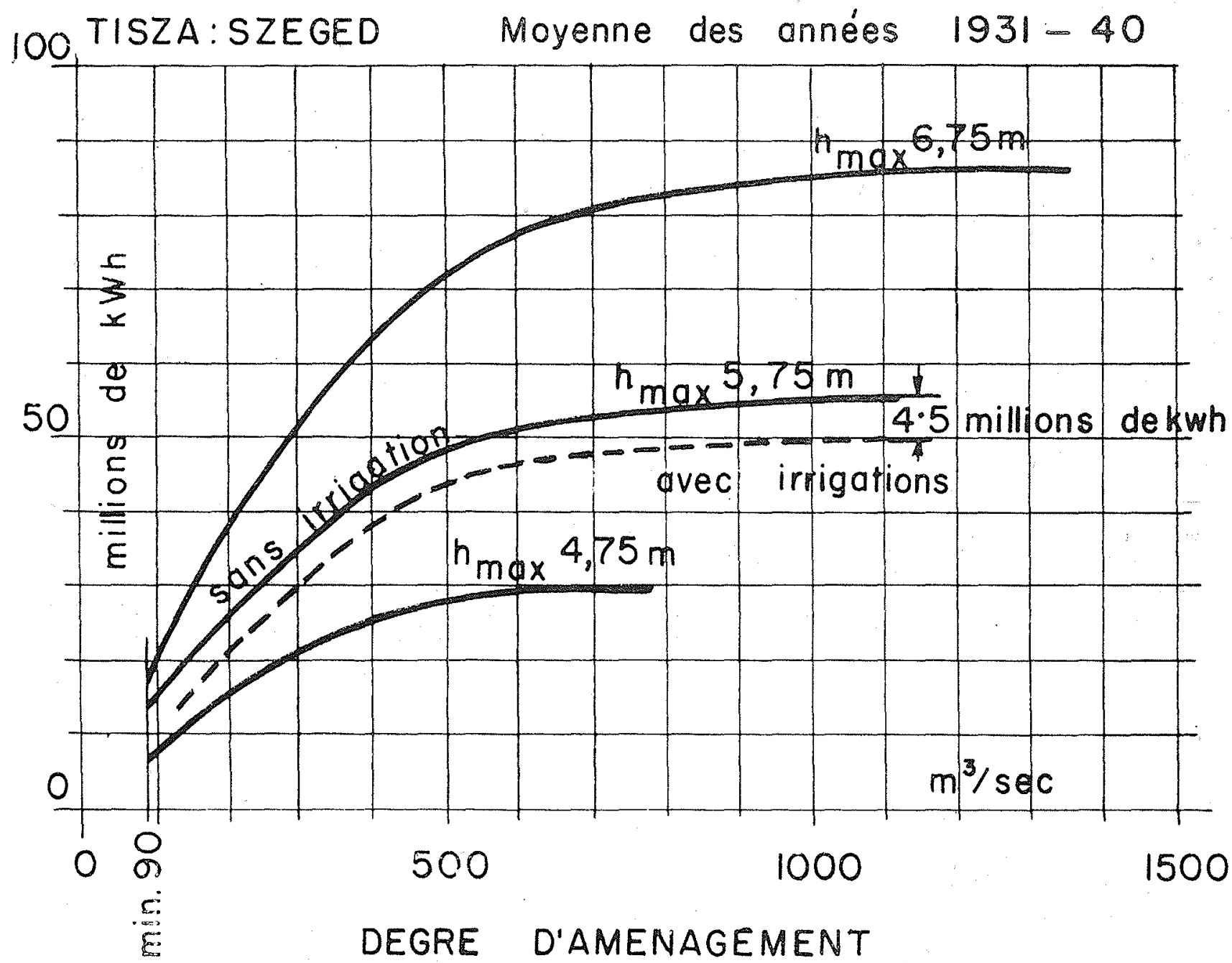

Fig. 6 
de canalisation de riviere, en raison de la hauteur fixce du niveau amont, la chute utilisable en lemps de hautes eaux, diminue et la production cesse si la hatuleur d'eau dépasse un certain niveau.

En prenant en considération tous ces facteurs, admettant les débits de $50 \%$, comme débit d'aménagement, la production possible d'énergie peut ètre évaluée à 2000 millions de kuh par an, maximum théorique correspondant au degré de (léveloppement des sciences techniques d'aujourd'hui qu'on ne pourrait pas pratiquement atteindre.

Si nous considérons que la canalisation de la Tisza s'impose dans tous les cas, en vue d'assurer les besoins en eau des irrigations tout en satisfaisant simultanément les intérêts de la navigation et que, par conséquent, les frais de constructions des barrages et des écluses ne grèvent pas la production d'énergie hydraulique, le prix de revient de l'énergie pouvant être produite sur la Tisza (150 millions de $\mathrm{kwh} / \mathrm{an}$ ) ne dépasse pas 18 centimes suisses/ $k w h, y$ compris les dépenses d'exploitation et d'amortissement. Ce prix de revient est done inférieur au prix de l'énergie thermique produite sur place.

D’après nos calculs les plus récents, les usines prévues sur le Danube seraient également à mòme de fournir de l'énergie à un prix modéré. Pa: exemple, le prix de revient de l'énergie produite par une usine de 375 millions de $\mathrm{kwh} / \mathrm{an}$ est évalué à 15-20 centimes suisses/kwh environ. Il $y$ a lieu de noter qu'en évaluant ce prix de revient, on a egalement fait compte des frais de barrages et des écluses nécessitées par la navigation. Il est surprenant que la production d'énergie des usi-nes prévues sur le Danube soit en état de soutenir la concurrence avec les usines de la Tisza alors que l'on a chargé les premieres des frais de construction de plusieur's ouvrages d'art. L'explication en est que les conditions hydrographiques du Danube sont beaucoup plus favorables (hautes eaux de plus courte durée, production plus équilibrée d'énergie) que celles de la Tisza.

En evaluant les frais de production de l'énergie, nous avons fait les calculs en admettant aussi bien dans le cas des usines de la Tisza que du Danube un amortissement de quarante ans et un taux d'intérêt de $4 \%$. En ce qui concerne les frais d'exploitation, nous nous sommes servis des expériences faites à l'Etranger.

En somme, nous évaluons la quantité d'énergie pouvant etre produile sur nos cours d'eau, it un prix raisonnable, it 1009 millions de liwh.

Considérant le besoin en énergie du pays qui va toujours en croissant, la rédaction des projels se rapportant a l'exploitation de la puissance précitée, mérite une altention spéciale. Le projet de trente ans du Ministere Hongrois de l'Agriculture prévoit la réalisation des aménagements suivants :

1"- Usines sur la Tisza

(4 biefs) production

annuelle ....... 150 millions de $\mathrm{kwh}$

2" Usines sur les cours

d'eau de moindre im-

portance (Sio, Raba

Zala, Sed, Hernad,

etc.) ......... 50 millions de $\mathrm{kwh}$

3"- Usines sur le Danube. 375 millions de kwh

$$
\text { Av TOtal .... } 575 \text { millions de kwh }
$$

(iig. 3 à 6$)$.

Les fig. 3 à 6 représentent les diagrammes se rapportant à la puissance et à la production annuelle d'énergie correspondant aux divers débits d'aménagement et aux maxima de chute pour une usine sur le Danube (fig. 3 et 4 ) et pour une usine sur la Tisza (fig. 5 et 6 ).

En cas de $q_{; i n}=2200 \mathrm{~m}^{3} / \mathrm{sec}, \quad h_{\text {mix }}=6.20$ et d'une puissance installée de $57.000 \mathrm{kw}$, on obtient sur le Danube 375 millions de kwh. L'emplacement de l'usine et la hauteur de la retenue ne sont pas encore fixés.

Les quatre usines sur la Tisza de maxima de chute de $6.20,5.00,5.00$, et 5.50 représenteraient au total une puissance mécanique de $35.000 \mathrm{kw}$ et même dans le cas où nous déduisons les volumes d'eau exigés par les irrigations, il reste encore une production annuelle d'énergie de 150 millions de kwh. 
Les graphiques se rapportant aux puissances démontrent des variations sensibles, voire l'interruption complète de la production en temps de hautes eaux, ce qui est manifestement défavorable. Mais étant donnée la diversité exis. tant dans le régime du Danube et de la Tisza et considérant que les usines hydrauliques dans le réseau national collaboreront avec les usines thermiques et que les usines hydrauliques déjà existantes ne fournissent que les $2 \%$ de la production totale d'énergie électrique du pays, les objections faites dans le passé contre l'aménagement des forces hydroélectriques ne pourraient plus être invoquées.

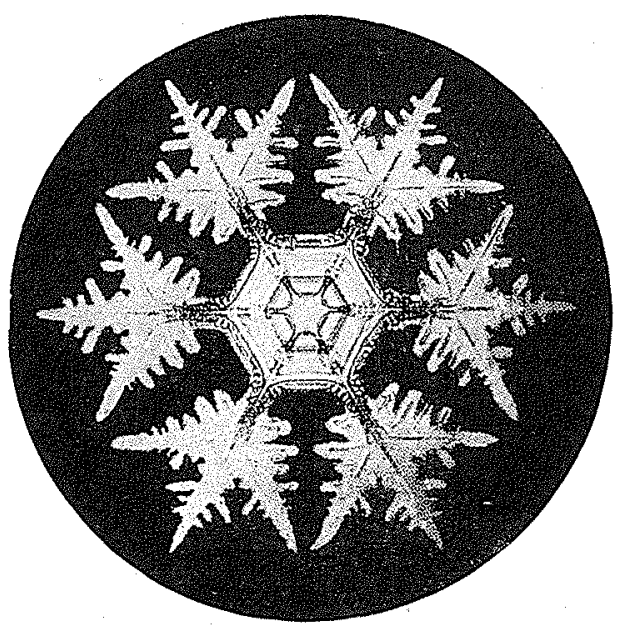

